\title{
INVESTIGACIONES
}

\section{CONOCIMIENTO QUE POSEEN LOS ESTUDIANTES DE PEDAGOGIA EN DIFICULTADES DE APRENDIZAJE DE LAS MATEMATICAS (DAM)}

\author{
Pedagogy students awareness on learning disabilities of mathematics (DAM) \\ Miguel Friz Carrillo, Susan Sanhueza Henríquez, Alejandra Sánchez Bravo \\ Departamento Ciencias de la Educación, Universidad del Bío-Bío, \\ Avenida La Castilla s/n, casilla 447, Chillán, Chile. \\ mfriz@ubiobio.cl
}

\section{Resumen}

La investigación tiene como propósito evaluar el conocimiento que poseen los estudiantes de pedagogía básica y educación parvularia acerca de las dificultades de aprendizaje en matemáticas (DAM). La investigación se adscribe a un enfoque cuantitativo, no-experimental descriptivo de tipo encuesta. El análisis de los datos se realizó a través del programa estadístico SPSS 14.0 y las técnicas utilizadas fueron principalmente estadísticos descriptivos de tendencia central (media) y dispersión (desviación típica), cálculo de frecuencias, porcentajes y la prueba $t$ para la comparación de medias entre grupos. Los resultados indican que si bien existe un conocimiento de los estudiantes en esta materia, aún resulta insuficiente para desarrollar competencias que les permitan intervenir positivamente en el aula.

Palabras clave: dificultades, aprendizaje, matemática, intervención educativa, formación inicial.

\begin{abstract}
The purpose of this study is to evaluate the awareness of students of elementary school teaching and nursing school teaching of learning disabilities of mathematics (DAM). The research adscribes a quantitative, nonexperimental and descriptive approach survey. Data were analyzed through SPSS 14.0 and the techniques were mainly descriptive statistics of medium tendency and dispersion (typical deviation), frequency calculus, percentage and the $t$ test for comparing media among the different groups. Results show that even though students have some knowledge on this matter, it is not enough so that to develop competences that will allow them a positive intervention in the classroom.
\end{abstract}

Key words: disabilities, learning, mathematics, educative intervention, starting abilities (formation). 


\section{INTRODUCCION}

Las teorías psicológicas nos proporcionan importantes aportes con respecto al rol fundamental que juegan las matemáticas como forma de aproximación a cualquier tipo de conocimiento y como base para ir adquiriendo otros aprendizajes más complejos. Sin embargo, si bien se reconoce su importancia, el fracaso en la enseñanza y el aprendizaje de las matemáticas tiene una alta prevalencia, convirtiéndose incluso en un filtro selectivo dentro del propio sistema educativo (Defior 2000). Estudios que intentan explicar el fracaso escolar (Lozano 2003) lo hacen partiendo de las variables que intervienen en la educación: padres (determinantes familiares), alumnos (determinantes personales) y profesores (determinantes académicos). Es precisamente esta última línea la que interesa profundizar en el presente estudio, ya que las dificultades que tienen los alumnos para aprender matemáticas en muchas ocasiones están vinculadas a situaciones didácticas inapropiadas o a la ausencia del conocimiento disciplinar de los profesores a quienes subyacen prácticas instrumentalizadas, centradas en la memorización bajo la idea de que un mismo dispositivo didáctico es posible de "aplicar" a toda la clase. Estas creencias son traspasadas de generación en generación y de esta manera el sistema universitario sólo termina reproduciendo estas prácticas, proporcionando a sus estudiantes escasos conocimientos relativos a cómo intervenir frente a las diferencias en el aula e involucrándose poco en implementar este tipo de formación en los planes de estudio. Desde esta perspectiva, el supuesto del que parte el trabajo es que lo que ocurra con estos profesores en formación debiera impactar significativamente en los aprendizajes transferidos posteriormente al aula (Friz 2007).

Este escenario genera nuevas demandas para la Educación Superior, particularmente en la formación inicial de profesores, para lo cual será necesario innovar en los programas de estudio, incorporando contenidos que resulten relevantes y pertinentes. Si bien existe un acuerdo en la incorporación de materias que tiendan a favorecer competencias para intervenir pedagógicamente en niños con NEE, las evidencias indican que los maestros en formación no se encuentran preparados para abordar la diversidad del aula y desconocen las opciones de apoyo específico que proporcionan los centros escolares. Una de las explicaciones de la ausencia de competencias profesionales es proporcionada por Becerril (1999) y señala que los actuales planes de estudios no preparan para la complejidad de los sucesos acontecidos en el aula, existiendo un predominio del conocimiento informal, con ausencia del conocimiento psicopedagógico (López 1999) que, entre otros factores, contempla problemáticas asociadas a las dificultades de aprendizaje. Habitualmente, temáticas relacionadas con la diversidad educativa son abordadas superficialmente y con un escaso tiempo en los currículos de formación, lo que obstaculiza instalar competencias que posteriormente respalden el trabajo del profesor en el aula.

\section{DIFICULTADES DE APRENDIZAJE EN MATEMATICAS E IMPLICANCIAS SOCIOEDUCATIVAS}

Las mediciones nacionales e internacionales coinciden en que las matemáticas es una de las áreas más problemáticas en términos de rendimiento de los alumnos. Como muestra de la dificultad que presenta la asignatura de matemáticas para los alumnos ofrecemos los siguientes datos: 
a) Los resultados en la prueba SIMCE 2006 indican que un 39\% de los alumnos y alumnas se encuentran en un nivel inicial en matemáticas, lo que indica que alumnos de $4^{\circ}$ año básico recién están iniciando la comprensión de los números naturales, la realización de los cálculos simples, el estudio de las formas geométricas y el manejo de los conceptos básicos de la resolución de problemas (MINEDUC 2006).

b) El fracaso en el aprendizaje de las matemáticas se encuentra estrechamente vinculado a los procesos lingüísticos, por ejemplo, en la comprensión del enunciado de un problema. Estudios indican que la probabilidad de responder correctamente a una pregunta de matemáticas no estaría únicamente condicionada por las habilidades matemáticas de los alumnos, sino además por su familiaridad con los distintos formatos de preguntas, vale decir, del lenguaje (Ramírez 2002).

c) Por otro lado, el $25 \%$ de los estudiantes chilenos con mejor desempeño en esta área (percentil 75), se ubica en 449 puntos. Esto muestra que el puntaje sobre el cual está el $25 \%$ de mejor rendimiento en Chile no alcanza al puntaje promedio de la OCDE (MINEDUC 2007).

Como es posible evidenciar, el aprendizaje de las matemáticas es un área prioritaria dentro de las políticas educativas, por ello es necesario hacerse cargo de aquellos estudiantes que presentan DAM. En un intento por conceptualizar las DAM podemos señalar que se presentan preferentemente en procesos asociados al cálculo y la resolución de problemas. Si bien podemos encontrar diversas definiciones en la literatura especializada, para nosotros, las DAM implican un nivel de rendimiento académico en matemáticas por debajo de lo esperado de acuerdo con la edad cronológica y nivel de desarrollo mental de los alumnos, aun teniendo un CI medio y escolaridad regular. Este bajo rendimiento no puede ser atribuido a un déficit sensorial (Castejón 2002: 159). Podríamos señalar que existen diversos enfoques que explicarían estas dificultades, algunos más innatistas como los enfoques neurológicos o cognitivos atribuyen las dificultades principalmente al alumno; sin embargo, el trabajo se adscribe a un enfoque de carácter evolutivo-educativo (Miranda, Fortes y Gil 1998), lo que implica que éstas podrían tener un carácter transitorio y ser prevenidas o corregidas a través de una estimulación adecuada, principalmente en los primeros años. Asimismo, este enfoque nos permite sostener que un alto porcentaje de alumnos que presentan DAM no logran responder a las estrategias que utilizan sus profesores para enseñar matemáticas, porque estos últimos no recibieron una formación adecuada para atender formas, ritmos y estilos diversos de aprendizaje, lo que indicaría que el problema radica preferentemente en la escuela.

Es necesario considerar que las dificultades guardan relación con los niveles de escolarización de los alumnos, por lo tanto, en los primeros años se trata de estimular el desarrollo de funciones básicas vinculando todo tipo de experiencias a su entorno; en tanto, en educación general básica, recién una vez instalados los procesos de lectura, escritura y matemática básicos podemos intentar realizar un diagnóstico de las DAM; antes de ello, los alumnos no cuentan (o están en desarrollo) con las operaciones que conforman las bases para aprendizajes de mayor complejidad. Por lo tanto, en el nivel de educación parvularia se trabajan contenidos como números, orden, correspondencia, clases, a través de actividades de lógica, cálculo, medida, relaciones espaciales y temporales con el apoyo de diversos recursos como bloques lógicos, regletas, programas de ordenador, etc., ya que en estos años "los niños desarrollan procesos básicos de atención, 
percepción, memorización y diferentes procesos cognitivos y metacognitivos y aspectos sociopersonales como el autoconcepto" (García 2001:168). Por su parte, en educación general básica ya se trabaja el cálculo escrito y ello exige una mayor formalización para seguir unos pasos establecidos por diferentes reglas y algoritmos, todo ello en razón de las cuatro operaciones. Se incorporan actividades de estimación, redondeo, resolución de problemas, representaciones geométricas. En ambos casos, la actuación del profesor juega un rol fundamental en la autoestima de los alumnos, ya que las evidencias demuestran que existe una relación directa entre actitudes hacia matemáticas y rendimiento académico (Ramírez 2005).

Dentro de las dificultades de aprendizaje más recurrentes se encuentran las referidas al dominio de numeración, por ejemplo, la falta de habilidad para representar visual o simbólicamente o codificar numéricamente la información; también se ha podido establecer que las principales dificultades en resolución de problemas se encuentran asociadas a las habilidades lingüísticas como la comprensión de términos, conceptos y formas de comunicar el contenido. La alta frecuencia de errores procedimentales está relacionada con el desarrollo adecuado de los procesos perceptivos como las dificultades en la lectura de símbolos numéricos o signos. También son recurrentes los problemas de encolumnamiento que dejan ver falencias en la estimulación temprana de habilidades visoespaciales que impiden procesar la información adecuadamente. En menor escala se observan dificultades relacionadas con déficit atencionales, que obstaculizan el reconocimiento de signos matemáticos, el procesamiento auditivo que interviene en la decodificación de información, en la memoria y en el ámbito motor. En síntesis, una adecuada intervención pedagógica implicará entre otros aspectos el conocimiento de la normativa que regula las NEE y las disposiciones que regulan la diversificación del currículo escolar, así como también que los profesores vuelvan a repensarse sus creencias sobre la enseñanza, sobre el aprendizaje y sobre la evaluación, considerando que hay dificultades de aprendizaje de las matemáticas generales y específicas, que requieren del conocimiento acerca de los efectos de un tipo de entrenamiento (García 2001:163).

\section{FORMACION DE PROFESORES PARA ATENDER LA DIVERSIDAD EDUCATIVA}

De acuerdo con los antecedentes expuestos y una vez conocida la importancia del aprendizaje de las matemáticas y las principales dificultades que presentan los alumnos en esta área, es necesario valorar si los profesores en formación cuentan con el conocimiento necesario para dar respuesta a estas problemáticas. Una revisión general de los programas de estudio de las carreras de pedagogía en Chile da cuenta de que en la mayoría de ellos sólo se registran una o dos asignaturas orientadas a la diversidad en ámbitos educativos, lo que reafirma que "las deficiencias que afectan a la formación de profesores son el resultado de planes de estudios sobrecargados de asignaturas, muchas de ellas innecesarias y un exceso de contenidos teóricos en detrimento de los prácticos" (González 2006:19). Las primeras aproximaciones de los estudiantes de pedagogía con el alumnado que presenta DAM en las escuelas se realizan a través de las prácticas pedagógicas, sin embargo, muchas de ellas son prácticas tempranas y los estudiantes aún no tienen conocimiento formal en esta materia. Si bien conocen los planes y programas de estudios oficiales, lo que develaría un cierto dominio en el área curricular, ellos manifiestan desconocer cómo abordar pedagógicamente las dificultades que tienen los niños en 
el aula. (Sanhueza 2006). Al respecto, existen diversas teorías acerca de qué es ser buen profesor o profesora, y en consecuencia existen distintos tipos de prácticas pedagógicas que responden a estas caracterizaciones. Asimismo, existen evidencias respecto de que los procedimientos, mecanismos o estrategias utilizados en la formación inicial son transferidos posteriormente a los estudiantes, entrando en conflicto modelos de formación en la época escolar, luego universitaria y conjuntamente con maestros del sistema educacional con quienes se vinculan a través de las prácticas iniciales. Un ejemplo lo constituye el resultado de una investigación reciente realizada en Chile (Latorre 2004) y que concluye señalando que profesores y profesoras que se inician profesionalmente, a la hora de "hacer clases" tienden a refugiarse en sus imágenes previas (de los profesores que tuvieron ellos mismos siendo alumnos en la escuela), o se someten inmediatamente a las demandas del contexto escolar en el que deben trabajar, es decir, "aprenden" en su temprana experiencia docente, lo que hace suponer que des-aprenden lo aprendido en la formación inicial o re-aprenden experiencialmente a través de su propia práctica a ser profesores. De esta forma, entran en contradicción creencias, percepciones y formas de actuación. Ante esta situación, el profesor en formación debe buscar un cuerpo de conocimientos artesanales que justifiquen su forma de actuar llevándolo a pensar equivocadamente que la experiencia es lo que más cuenta (Gilroy 1993). En esta línea, la literatura nos indica que "muchos profesores y profesoras manifiestan una aparente simplicidad conceptual y de pensamiento en general, simplicidad que se caracteriza por un pensamiento básicamente intuitivo, no racional, con escasez de términos abstractos, actitudes dogmáticas, decisiones tomadas impulsivamente, falta de indagación en las acciones, improvisación y justificación basada en la experiencia” (Imbernón 2002).

Los antecedentes expuestos llevan a plantear la urgencia de mejorar la formación inicial de profesores, incorporando contenidos programáticos que vayan en esta línea y sobre todo favoreciendo actitudes de aceptación de las diferencias. Tal como reportan investigaciones, el profesorado necesita conocer formas más flexibles y versátiles de organizar el funcionamiento del aula, formas capaces de promover el uso de estrategias adaptativas generales eficaces para todos los alumnos (Cardona 2002). Como se puede apreciar, el tipo de conocimiento que incorporen los profesores juega un papel fundamental en el posterior ejercicio de la profesión, por lo tanto, lo que debe conocer el profesor está íntimamente relacionado con los contextos y situaciones en los que vaya a utilizar su conocimiento, es decir, con contextos y situaciones de enseñanza de las matemáticas que han de ser tenidos en cuenta en los programas de formación (Llinares 1994). En síntesis, las DAM demandan una formación inicial de profesores sustentada en un enfoque integrado, en donde las matemáticas no sean ajenas a la vida cotidiana, sino parte de ella y que en consecuencia requieren para su aprendizaje la condición de un saber situado.

A partir de los antecedentes expuestos en las páginas que preceden, cabe preguntarse: ¿Los estudiantes de pedagogía cuentan con herramientas formativas que les permitan intervenir educativamente en alumnos con dificultades de aprendizaje en matemáticas? $¿$ Conocen los dominios en los cuales se presentan con mayor frecuencia las dificultades de aprendizaje? ¿Conocen las repercusiones de sus decisiones en la autoestima de alumnos con dificultades de aprendizaje de matemáticas?

Las interrogantes planteadas pueden concretarse en el siguiente propósito de investigación que es analizar el conocimiento que poseen los estudiantes de pedagogía para atender a alumnos con dificultades de aprendizaje en matemáticas (DAM), cuyos objetivos específicos son: 
1. Analizar el conocimiento de los estudiantes de pedagogía relativo a los dominios en los cuales se presentan preferentemente las Dificultades de Aprendizaje en Matemáticas (DAM).

2. Analizar el conocimiento de los estudiantes de pedagogía relativos a los efectos en la conducta socioeducativa del alumnado que presenta DAM.

3. Conocer las actitudes de los estudiantes de pedagogía respecto a la atención de los alumnos que presentan dificultades en el aprendizaje de las matemáticas (DAM).

4. Examinar el tipo de adaptaciones al currículum que proponen con mayor frecuencia los estudiantes de pedagogía para la atención de alumnos con DAM.

5. Analizar diferencias estadísticamente significativas de conocimiento y actitud de los estudiantes de pedagogía en función de su especialidad.

\section{METODO}

Enfoque y diseño. La investigación se adscribe a un enfoque cuantitativo, específicamente un diseño no-experimental descriptivo del tipo encuesta, en coherencia con el problema en estudio (Gay y Airasian 2000). Así, la información relativa al conocimiento en Dificultades de Aprendizaje de las Matemáticas se recaba a través de un cuestionario dirigido a los estudiantes de pedagogía.

Selección y composición de la muestra. El estudio fue realizado en una Universidad regional de Chile, de carácter público. Para ello se realizó un muestreo no probabilístico de tipo intencional a dos cortes de alumnado en el período académico 2006 (78,6\% del total de la promoción) y 2007 (78,9\% del total de la promoción); constituyendo una muestra total de 145 estudiantes de pedagogía, de los cuales 88 (61\%) estudiaban educación básica y $57(39 \%)$ educación parvularia. Las edades de los participantes fluctuaban entre los 20 y 28 años, siendo el promedio 22 años. En cuanto al género, predominan las mujeres $(n=122 ; 84,1 \%)$ sobre los varones $(n=23 ; 15,9 \%)$. Ya que el muestreo fue intencional se estableció como criterios a priori que los participantes debían ser estudiantes de último año de formación de las carreras de educación parvularia o educación básica, que contaran con algún tipo de experiencia previa a través de las prácticas pedagógicas en los centros educativos y que tuvieran algún tipo de formación previa en la temática de necesidades educativas especiales.

Variables de investigación e instrumento. Los datos del estudio fueron recogidos a través de una encuesta de opinión dirigida a los estudiantes de pedagogía acerca del conocimiento en dificultades de aprendizaje de las matemáticas (DAM) que se compone de tres partes. En la primera, se solicita información acerca de variables que ilustran la muestra como son titulación de los estudiantes de pedagogía, edad y género. En la segunda parte se presentan 18 ítemes referidos a dominios matemáticos, conducta socioeducativa $\mathrm{y}$ actitudes de los estudiantes frente a los alumnos con DAM. Estas cuestiones fueron informadas a través de una escala tipo Lickert en la que debieron marcar su grado de acuerdo desde 1 (Muy en desacuerdo) a 4 (Muy de acuerdo); en consecuencia, las puntuaciones igual o mayores que 3 mostrarían un nivel aceptable de conocimiento en la materia. Finalmente, el último apartado fue construido a partir de una pregunta abierta 
que grafica una situación de suma con reserva en la cual el resultado proporcionado por un alumno muestra errores en el aprendizaje matemático pudiendo ser explicados a partir de fallas en el procesamiento de la información, errores en el valor posicional de los números, dificultades con situaciones de canje, entre otros. Las respuestas obtenidas fueron codificadas a partir de los elementos del currículum en los cuales los estudiantes realizaron preferentemente adaptaciones. En síntesis, las variables quedan organizadas de la siguiente manera:

I. Variables de tipo demográfico.

II. Variables relativas a los dominios en que se presentan las dificultades de aprendizaje en matemáticas (DAM).

III. Variables relativas a los efectos en la conducta socioeducativa del alumnado que presenta dificultades de aprendizaje en matemáticas (DAM).

IV. Variables relativas a las actitudes de los estudiantes de pedagogía respecto a la atención de los alumnos que presentan dificultades en el aprendizaje de las matemáticas (DAM).

V. Variables relativas a las formas de actuación de los estudiantes de pedagogía referidos al currículum.

Para la elaboración de la encuesta se recurrió a la revisión de literatura especializada dotando de este modo de significatividad a los ítemes que componen el instrumento. Posteriormente, el instrumento se sometió a valoración de expertos cuyas aportaciones indican un I.V.C (Indice de validez de contenido) correspondiente a 0.93. Tras un primer análisis para estimar la fiabilidad del instrumento se obtuvo un coeficiente Alpha de Cronbach de .874 , lo cual indica que el instrumento posee una buena consistencia interna (grado en que los ítemes de la escala miden el constructo que se desea medir), es decir, la información es considerada fiable.

Procedimiento para el análisis de los datos. La recogida de datos se llevó a cabo de manera grupal durante una hora de clases. El encuestador informó a los estudiantes acerca del objetivo de la investigación y solicitó el consentimiento para su aplicación. No se estableció medición de tiempo para las respuestas y cada participante dio lectura individual respondiendo de acuerdo con sus preferencias. El análisis de los datos se realizó a través del programa estadístico SPSS 14.0 y las técnicas utilizadas fueron principalmente estadísticos descriptivos de tendencia central (media) y dispersión (desviación típica) y cálculo de frecuencias y porcentajes. Sólo en el caso del último objetivo que pretendía buscar diferencias estadísticamente significativas a partir de la titulación de los sujetos se utilizó la prueba $t$ (muestras independientes) para la comparación de medias entre grupos trabajándose en este caso con un $\alpha=.05$.

\section{RESULTADOS}

Los valores medios y las desviaciones estándar de las variables dependientes para el total de la muestra $(N=145)$, así como los porcentajes de respuestas por ítemes figuran en las tablas 1, 2, 3 y 4. Todos estos elementos sirven de base al análisis de los resultados clasificado por objetivos que se presenta a continuación. 
Conocimiento de los estudiantes de pedagogía relativos a los dominios en que se presentan las dificultades de aprendizaje en matemáticas (DAM).

Tabla 1

Dominios en que se presentan preferentemente las DAM

\begin{tabular}{|l|c|c|c|c|c|c|}
\hline & $\underline{\mathrm{M}}$ & $\underline{\mathrm{DT}}$ & $\begin{array}{c}\mathrm{MD} \\
\%\end{array}$ & $\begin{array}{c}\mathrm{D} \\
\%\end{array}$ & $\begin{array}{c}\mathrm{A} \\
\%\end{array}$ & $\begin{array}{c}\mathrm{MA} \\
\%\end{array}$ \\
\hline Numeración & 2.35 & .73 & 4,1 & $\underline{67.6}$ & 17,2 & 11 \\
\hline Operatoria & 2.72 & .69 & 8,3 & 16,6 & $\underline{69,7}$ & 5,5 \\
\hline Procesos perceptivos & 2.84 & .85 & 10,3 & 14,5 & $\underline{55,9}$ & 19,3 \\
\hline Resolución Problemas & 3.74 & .63 & 2,1 & $4 ., 1$ & 11 & $\underline{82,8}$ \\
\hline Funciones Básicas & 3.19 & .89 & 2,8 & 23,4 & 25,5 & $\underline{48,3}$ \\
\hline
\end{tabular}

De acuerdo con los resultados, la mayoría de los encuestados $(M=3.74 ; 82,8 \%)$ estuvo muy de acuerdo en que las DAM se presentan preferentemente en el dominio de la Resolución de Problemas. No obstante, un porcentaje significativo de estudiantes estuvo de acuerdo en que las dificultades en el aprendizaje de las matemáticas además se relacionarían con la adquisición de las Funciones Básicas $(M=3.19 ; 48,3 \%)$; los Procesos Perceptivos $(M=2.84 ; 55,9 \%)$ y la Operatoria $(M=2.72 ; 69,7 \%)$. Con respecto al dominio Numeración, la mayoría de los encuestados $(M=2.35 ; 67,6 \%)$ consideró que este ámbito no reporta mayores dificultades para su enseñanza-aprendizaje.

Conocimiento de los estudiantes de pedagogía relativos a los efectos en la conducta socioeducativa del alumnado que presenta DAM.

Tabla 2

Conocimiento de los estudiantes de pedagogía relativos a la conducta de los alumnos que presentan DAM

\begin{tabular}{|l|c|c|c|c|c|c|}
\hline & $\underline{\mathrm{M}}$ & $\underline{\mathrm{DT}}$ & $\begin{array}{c}\mathrm{MD} \\
\%\end{array}$ & $\begin{array}{c}\mathrm{D} \\
\%\end{array}$ & $\begin{array}{c}\mathrm{A} \\
\%\end{array}$ & $\begin{array}{c}\mathrm{MA} \\
\%\end{array}$ \\
\hline Busca la ayuda del profesor & 1.79 & .91 & $\underline{49,7}$ & 26,9 & 18,6 & 4,8 \\
\hline Se ubica lejos del profesor en la sala de clases & 3.47 & .89 & 9 & - & 26,2 & $\underline{64,8}$ \\
\hline Se esfuerza en terminar sus deberes escolares & 2.81 & .86 & 2,8 & $\underline{40}$ & 30,3 & 26,9 \\
\hline Presenta problemas de disciplina & 2.71 & .71 & 4,8 & 29,7 & $\underline{55,2}$ & 10,3 \\
\hline Presenta habilidades de liderazgo & 2.10 & .60 & 13,8 & $\underline{62,8}$ & 23,4 & - \\
\hline Manifiesta ansiedad frente a la evaluación & 3.50 & .65 & - & 9 & 32,4 & $\underline{58,6}$ \\
\hline Manifiesta una actitud proactiva en el aula & 2.46 & 1.11 & $\underline{26,2}$ & 24,8 & 25,5 & 23,5 \\
\hline
\end{tabular}


La mayoría de los encuestados estuvo muy de acuerdo en que los alumnos que presentan DAM no solicitan ayuda al profesor para resolver sus dificultades $(M=1.79$; $49,7 \%)$, sólo un porcentaje menor $(23,4 \%)$ opinó lo contrario. Concordante con lo anterior, un alto porcentaje de encuestados opina que los alumnos con DAM, por lo general, prefieren ocupar un puesto de trabajo distante al escritorio del profesor $(M=3.47 ; 64,8 \%)$. Con respecto al cumplimiento de los deberes escolares la mayoría de los encuestados consideró que los alumnos con DAM no se esfuerza en terminar sus deberes escolares $(M=2.81 ; 40 \%)$, sin embargo, si observamos en detalle los datos podemos ver que un grupo significativo estuvo de acuerdo y muy de acuerdo en que los alumnos se esfuerzan por completar los deberes asignados $(57,2 \%)$. Por otra parte, la mayoría de los participantes estuvo de acuerdo en que los alumnos con DAM presentan problemas de disciplina $(M=2.71 ; 55,2 \%)$, frente a un $29,7 \%$ que manifestó su desacuerdo. Además, la mayoría de los encuestados consideró que este tipo de alumnado no presenta habilidades de liderazgo $(M=2.10 ; 62,8 \%)$, sólo un $23,4 \%$ considera que las podría presentar. También la mayoría estuvo muy de acuerdo en que los alumnos con DAM manifiestan ansiedad frente a una situación de evaluación $(M=3.50 ; 58,6 \%)$ en oposición a un $9 \%$ que consideró que no la presenta. Por último, con respecto a la conducta proactiva que podría mostrar un alumno con DAM, la respuesta de los encuestados se divide en forma homogénea, un grupo correspondiente al 51\% consideró que el alumno no presenta una conducta proactiva frente a un $49 \%$ que consideró que sí la presenta.

Actitudes de los estudiantes de pedagogía respecto a la atención de los alumnos que presentan dificultades en el aprendizaje de las matemáticas (DAM).

En la tabla 3 se presentan los datos relativos a las actitudes que manifiestan los estudiantes de pedagogía con respecto a la atención educativa de los alumnos y alumnas que presentan dificultades de aprendizaje y en los cuales se basan los resultados que presentamos a continuación.

\section{Tabla 3}

Actitud que manifiestan los estudiantes de pedagogía hacia el alumnado que presenta DAM

\begin{tabular}{|l|c|c|c|c|c|c|}
\hline & $\underline{\mathrm{M}}$ & $\underline{\mathrm{DT}}$ & $\begin{array}{c}\mathrm{MD} \\
\%\end{array}$ & $\begin{array}{c}\mathrm{D} \\
\%\end{array}$ & $\begin{array}{c}\mathrm{A} \\
\%\end{array}$ & $\begin{array}{c}\mathrm{MA} \\
\%\end{array}$ \\
\hline Atención individualizada (Dentro del aula regular) & 3.06 & .88 & 7,6 & 13,8 & $\underline{44,1}$ & 34,5 \\
\hline Evaluación diferenciada & 3.66 & .65 & 2,1 & 3,4 & 21,4 & $\underline{73,1}$ \\
\hline Derecho a una educación de calidad & 3.83 & .49 & - & 4,8 & 7,6 & $\underline{87,6}$ \\
\hline Apoyo de grupo diferencial (Fuera del aula regular) & 3.27 & .98 & 9 & 11 & 24,1 & $\underline{55,9}$ \\
\hline Colaboración multidisciplinar & 2.36 & .90 & 15,2 & $\underline{47,6}$ & 23,4 & 13,8 \\
\hline Promoción & 2.68 & .83 & 7,6 & 33,1 & $\underline{42,8}$ & 16,6 \\
\hline
\end{tabular}


La gran mayoría de los encuestados estuvo muy de acuerdo $(M=3.83 ; 87,6 \%)$ en que los alumnos y alumnas que presentan DAM tienen derecho, al igual que todos los estudiantes, a recibir una educación de calidad. Sólo un porcentaje menor $(4,8 \%)$ de encuestados consideró que este derecho debe ser vulnerado al tratarse de un alumnado que requiere una mayor atención educativa. No obstante, para la mayoría de los futuros profesores la atención del alumnado que presenta dificultades de aprendizaje debe realizarse fuera de la sala de clases y bajo la responsabilidad de un especialista $(M=3.27$; $55,9 \%$ ), sólo un porcentaje menor estuvo de acuerdo en apoyar dentro de la sala de clases con una atención más individualizada la dificultad de aprendizaje del alumnado. Con respecto a la evaluación diferenciada, los encuestados estuvieron muy de acuerdo con la aplicación de esta estrategia $(M=3.66 ; 73,1 \%)$. No obstante, al tratarse de la promoción de curso, las opiniones se presentan divididas, mientras que el 59,2\% estuvo de acuerdo con la promoción un 40,7\% no lo estuvo.

\section{Intervención pedagógica de los estudiantes de pedagogía}

En la tabla 4 se presentan las frecuencias y porcentajes de respuestas para el total de la muestra $(N=145)$ relativos al tipo de intervención educativa que los encuestados proponen con mayor frecuencia para la atención de alumnos con DAM. Los resultados se presentan a continuación.

\section{Tabla 4}

Tipo de intervención educativa

\begin{tabular}{|l|c|c|c|}
\hline & Frecuencia & Porcentaje & $\begin{array}{c}\text { Porcentaje } \\
\text { acumulado }\end{array}$ \\
\hline Adaptación del contenido & 65 & 44,8 & 44,8 \\
\hline Recurso didáctico & 30 & 20,7 & 65,5 \\
\hline Estrategia cognitiva & 23 & 15,9 & 81,4 \\
\hline Metodología personalizada & 21 & 14,5 & 95,9 \\
\hline Estrategia de visualización & 3 & 2,1 & 97,9 \\
\hline Derivación profesional no docente & 3 & 2,1 & 100,0 \\
\hline Total & 145 & 145 & \\
\hline
\end{tabular}

Como se puede observar aproximadamente la mitad de los encuestados $(44,8 \%)$ utilizó una adaptación del contenido frente a una DAM. Un porcentaje menor de los estudiantes de pedagogía propuso recurrir al uso de material didáctico $(20,7 \%)$; estrategia cognitiva $(15,9 \%)$ o la aplicación de un trabajo más personalizado con el alumno (14,5\%). 
Diferencias de conocimiento y actitud de los estudiantes de pedagogía en función de su especialidad

Al analizar las respuestas medias de ambos grupos (tabla 5) en función de la especialidad (EP / EGB.), se pueden apreciar diferencias estadísticamente significativas.

\section{Tabla 5}

Comparación de medias en función de la especialidad

\begin{tabular}{|c|c|c|c|c|c|c|}
\hline & $\begin{array}{l}\text { Educ. } \\
\text { Parvularia } \\
\text { (EP) }\end{array}$ & $\begin{array}{c}\text { Educ. General } \\
\text { Básica } \\
\text { (EGB) }\end{array}$ & & & & \\
\hline $\begin{array}{l}\text { Conocimiento y actitud de los estudiantes } \\
\text { de pedagogía hacia las DAM. }\end{array}$ & $\underline{\mathrm{M} / \mathrm{DT}}$ & $\underline{\mathrm{M} / \mathrm{DT}}$ & $\underline{t}$ & $\mathrm{gl}$ & $\mathrm{p}$ & Dirección \\
\hline $\begin{array}{l}\text { Conocimiento en Dominios } \\
\text { Numeración }\end{array}$ & $2.23 / 1.01$ & $2.17 / .37$ & -3.29 & 66 & .002 & $\mathrm{P}>\mathrm{B}$ \\
\hline Operatoria & $2.65 / .76$ & $2.77 / .63$ & 1.01 & 103 & .315 & \\
\hline Percepción & $3.14 / .95$ & $2.65 / .72$ & -3.32 & 97 & .001 & $\mathrm{P}>\mathrm{B}$ \\
\hline Resolución de problemas & $3.49 / .88$ & $3.91 / .28$ & 3.43 & 63 & .001 & $\mathrm{P}<\mathrm{B}$ \\
\hline Funciones básicas & $2.91 / 1.02$ & $3.38 / .74$ & 2.94 & 94 & .004 & $\mathrm{P}<\mathrm{B}$ \\
\hline $\begin{array}{l}\text { Conocimiento en conducta del alumnado } \\
\text { Solicita ayuda }\end{array}$ & $2.49 / .90$ & $1.33 / .56$ & -8.64 & 83 & .000 & $\mathrm{P}>\mathrm{B}$ \\
\hline Se ubica cerca del profesor & $3.40 / .67$ & $3.51 / 1.00$ & .77 & 142 & .442 & \\
\hline Termina sus deberes & $3.25 / .80$ & $2.53 / .78$ & -5.23 & 117 & .000 & $\mathrm{P}>\mathrm{B}$ \\
\hline Presenta problemas de disciplina & $2.61 / .84$ & $2.77 / .62$ & 1.22 & 94 & .223 & \\
\hline Presenta habilidades de liderazgo & $2.19 / .69$ & $2.03 / .53$ & -1.47 & 98 & .145 & \\
\hline Presenta ansiedad & $3.19 / .66$ & $3.69 / .57$ & 4.65 & 106 & .000 & $\mathrm{P}<\mathrm{B}$ \\
\hline Actitud proactiva & $1.84 / .77$ & $2.86 / 1.12$ & 6.46 & 142 & .000 & $\mathrm{P}<\mathrm{B}$ \\
\hline $\begin{array}{l}\text { Actitud hacia el alumnado } \\
\text { Atención dentro del aula regular }\end{array}$ & $3.14 / 1.00$ & $3.00 / .80$ & -.88 & 100 & .378 & \\
\hline Evaluación diferencial & $3.61 / .49$ & $3.68 / .73$ & .66 & 142 & .507 & \\
\hline Calidad educativa & $3.84 / .49$ & $3.82 / .49$ & -.28 & 119 & .776 & \\
\hline Atención fuera del aula regular & $2.56 / 1.11$ & $3.73 / .49$ & 7.41 & 70 & .000 & $\mathrm{P}<\mathrm{B}$ \\
\hline Derivación & $2.09 / 1.02$ & $2.53 / .77$ & 2.81 & 96 & .006 & $\mathrm{P}<\mathrm{B}$ \\
\hline Promoción de curso & $2.68 / .80$ & $2.68 / .86$ & -.01 & 125 & .986 & \\
\hline
\end{tabular}

Significancia al $1 \%$. 
Estas diferencias significativas se observan en tres de las variables evaluadas (Dominios en que se presentan las DAM, conducta socioeducativa del alumnado y actitud hacia el alumnado). De modo ilustrativo estas diferencias se pueden observar en las figuras 1, 2 y 3 respectivamente.

\section{Figura 1}

Dominios en que se presentan las DAM

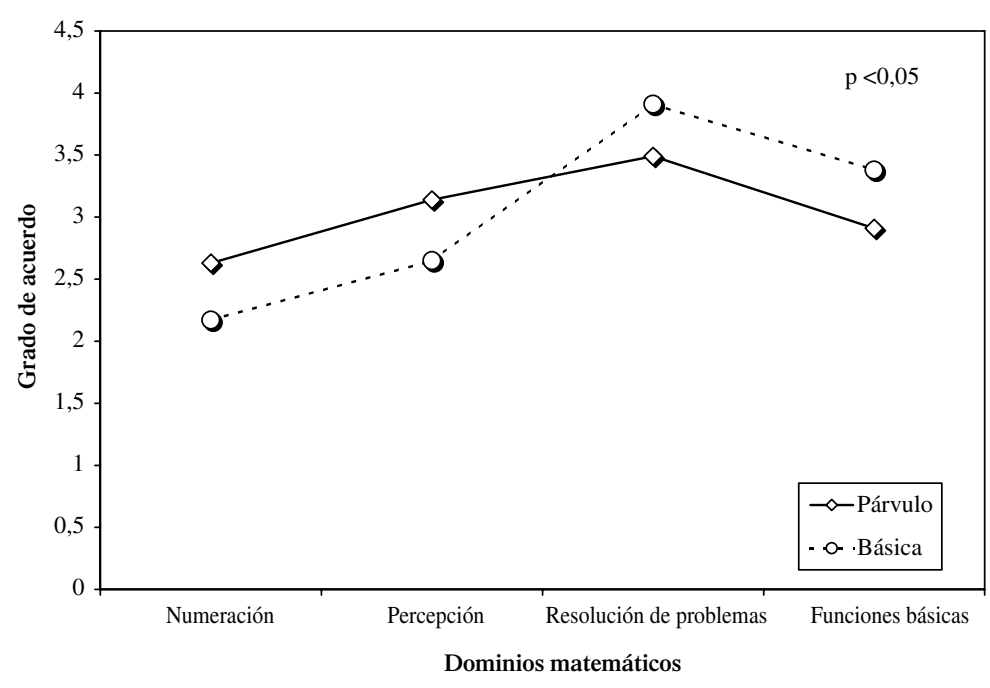

Figura 2

Conductas socioeducativas del alumno con DAM

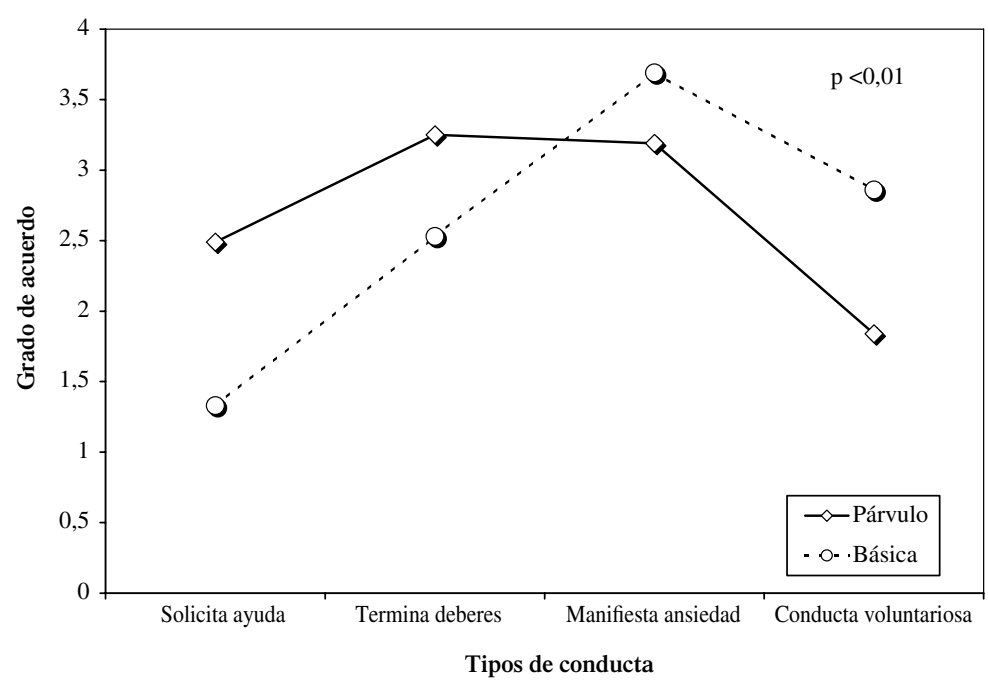


Figura 3

Actitud del profesor hacia e alumno

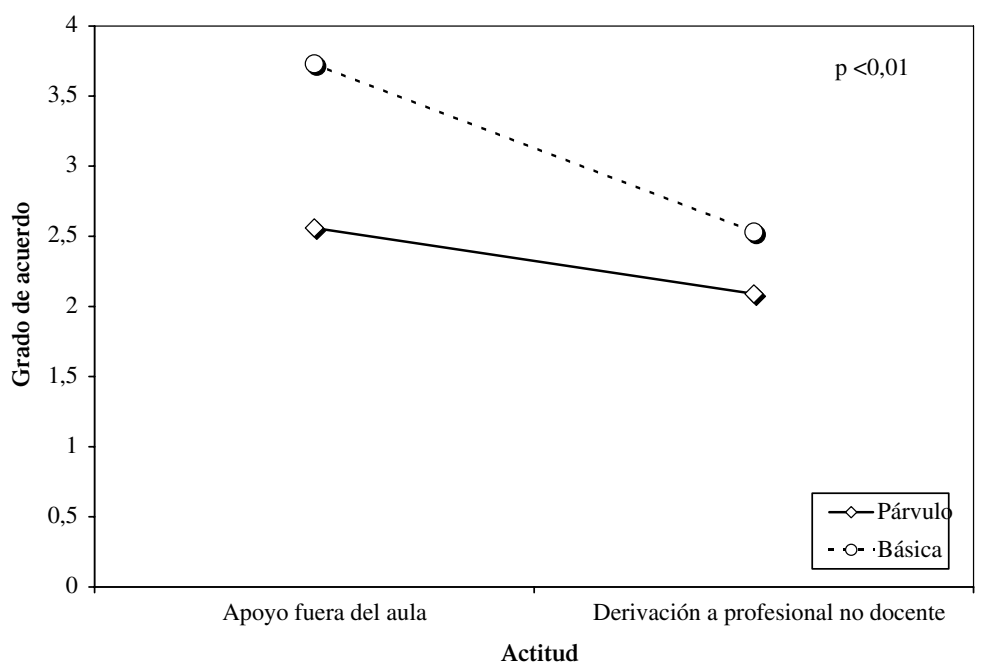

Dominios en que se presentan las DAM

Los estudiantes de EGB manifestaron estar muy de acuerdo en que las DAM se presentan preferentemente en el dominio Resolución de Problemas $[t(63)=3.43$; $\mathrm{p}=.001$ ] en relación a los estudiantes de EP que manifestaron un acuerdo. Además, los estudiantes de EGB mostraron un mayor grado de acuerdo en que las DAM se pueden presentar en el dominio Funciones Básicas $[t(94)=2.94 ; \mathrm{p}=.004]$ que los estudiantes de EP. No obstante, los estudiantes de EP estuvieron de acuerdo que las DAM podrían implicar dificultades de Percepción [ $t(97)=-3.32 ; \mathrm{p}=.001]$, mientras que las respuestas de los estudiantes de EGB no alcanzaron el acuerdo. Por último, los estudiantes de EP tienden a pensar que las DAM se relacionarían con dificultades en el dominio Numeración $[t(66)=-3.29 ; \mathrm{p}=.002]$.

\section{Conducta socioeducativa del alumno con DAM}

Los estudiantes de EGB mostraron un mayor grado de acuerdo en que los alumnos que presentan DAM manifiestan mayor ansiedad frente a la evaluación que los alumnos que no presentan tal dificultad $[t(106)=4.65 ; \mathrm{p}=.000]$ que los estudiantes de EP. Además, los estudiantes de EGB estuvieron en mayor grado de acuerdo en que los alumnos con DAM presentan una conducta proactiva frente al profesor $[t(142)=6.46 ; \mathrm{p}=.000]$, mientras que los estudiantes de EP mostraron desacuerdo al respecto. Por otra parte, los estudiantes de EP estuvieron de acuerdo en que los alumnos con DAM se esfuerzan en terminar sus deberes escolares $[t(117)=-5.23 ; \mathrm{p}=.000]$, en tanto los estudiantes de EGB no alcanzaron el acuerdo. Por último, los estudiantes de EP estuvieron en desacuerdo 
en que el alumno con DAM solicita ayuda al profesor para resolver las dificultades de aprendizaje que se le presentan $[t(83)=-8.64 ; \mathrm{p}=.000]$, mientras que los estudiantes de EGB estuvieron muy en desacuerdo.

\section{Actitud de los estudiantes de pedagogía hacia el alumnado con DAM}

Los estudiantes de EGB manifestaron en gran medida su acuerdo en que los alumnos que presentan DAM deben ser atendidos fuera de la sala de clases por un especialista $[t(70)=7.41 ; \mathrm{p}=.000]$, mientras que los estudiantes de EP se mostraron más bien en desacuerdo. Otra diferencia significativa se observa respecto a la derivación del alumno: los estudiantes de EP manifestaron en mayor grado su desacuerdo en derivar al alumno que presenta DAM a un profesional no docente para resolver el problema $[t(96)=2.81$; $\mathrm{p}=.006]$ que los estudiantes de EGB.

\section{DISCUSION Y CONCLUSIONES}

A partir de los resultados expuestos, es posible concluir que los estudiantes de pedagogía reconocen que el dominio en que se presentan preferentemente las DAM corresponde al de Resolución de Problemas, situación que se condice con los últimos reportes de la prueba SIMCE (Sistema de Medición de la Calidad de Enseñanza) aplicada en Chile. Es importante señalar que este dominio requiere de un desarrollo cognitivo adecuado y niveles de pensamiento más complejos que implican organizar información con miras a posibles soluciones en las cuales muchas veces el alumno debe inferir la operatoria apropiada y resolver el problema en un contexto más global para lograr finalmente aplicar y transferir a situaciones extracurriculares los conocimientos previamente adquiridos. Por otra parte, podemos concluir que para los futuros profesores el dominio Numeración es donde con menor frecuencia se presentarían las DAM, lo que lleva a suponer que los estudiantes consideran este dominio de fácil logro. Al respecto, la literatura especializada señala que la construcción del número es un aspecto de complejidad creciente, siendo las dificultades más recurrentes la inversión de números y las dificultades en el valor posicional. En general, es posible apreciar que los estudiantes de pedagogía se centran referentemente en un tipo de dificultad, desestimando otros problemas que los alumnos podrían presentar. Por lo tanto, pierden la perspectiva global de las DAM, situación que podría traer consecuencias muy desfavorables para el tratamiento educativo como ser un mal diagnóstico y el uso de estrategias educativas inadecuadas.

En relación con el conocimiento de los efectos en la conducta socioeducativa del alumnado que presenta dificultades de aprendizaje en matemáticas, es posible concluir que los estudiantes de pedagogía tienen conciencia de que el alumnado con dificultades de aprendizaje en matemáticas manifiesta una conducta particular que se podría caracterizar por una conducta de evitación hacia el profesor y mayor ansiedad frente a la evaluación que alumnos que no presentan problemas de aprendizaje. Frente a tal situación no debemos olvidar que somos los profesores los encargados de promover espacios de encuentro dentro del aula y crear un clima de confianza que favorezca el aprendizaje, evitando de este modo que el alumno se invisibilice dentro del grupo. Sin embargo, también podemos concluir que los futuros profesores dejan ver en sus respuestas estereotipos que perjudican 
aun más al alumno, como son la falta de disciplina, liderazgo e iniciativa, y sobre los cuales habría que trabajar, por cuanto estas actitudes son transferidas posteriormente al ejercicio profesional.

En cuanto a la actitud que manifiestan los estudiantes de pedagogía hacia el alumnado con DAM, es posible concluir que se presenta ambigua y en cierto grado contradictoria. Si bien la mayoría considera deben recibir educación de calidad, evaluación diferenciada y ser promovidos de curso, un número importante de ellos no está dispuesto a ofrecerla, ya que la mayoría está de acuerdo en que estos alumnos deben ser atendidos fuera de la sala de clases por un especialista. Por tanto, un importante número de estudiantes opta por "desligarse" de la responsabilidad, tiempo y compromiso que demanda una atención centrada en el alumno. Somos conscientes de que en muchas oportunidades esta conducta de delegar el apoyo a un especialista no sólo implica una actitud negativa, sino también oculta un desconocimiento de las estrategias que debe poner en práctica el profesor para atender a los alumnos con DAM.

Con respecto al tipo de intervenciones pedagógicas que preferentemente utilizaron los estudiantes de pedagogía frente a una DAM, es posible concluir que principalmente recurren a la adaptación de contenido, mayoritariamente proponen graduaciones por complejidad, complementación y selección de contenidos que consideran relevantes. Esto nos lleva a pensar que en su formación ha prevalecido un enfoque curricular normativo centrado en la transmisión de contenidos. Este razonamiento además se justifica por la escasa prevalencia de respuestas que promuevan la diversificación curricular y las distintas formas de aprender en otros elementos del currículum que también son importantes.

Por último, podemos concluir que existen diferencias estadísticamente significativas en las respuestas proporcionadas por los estudiantes de ambas titulaciones principalmente relacionadas con los dominios en que se presentan las DAM y la conducta socioeducativa que podría presentar este alumnado. Creemos que estas diferencias podrían explicarse por diferencias del perfil que caracteriza a cada carrera, por cuestiones de formación y de experiencias prácticas.

\section{Implicancias educativas}

Luego de finalizado el estudio y analizados en profundidad los resultados, creemos que la formación inicial docente, cuya responsabilidad le compete principalmente a las universidades, debe contemplar estas problemáticas e incorporarlas de manera transversal al currículo de formación. No sólo importa adquirir conocimientos específicos, que sin duda mejoran la práctica docente, sino además desarrollar una actitud positiva hacia el alumnado que mayor atención requiere, sensibilizando y motivando a los futuros docentes en prácticas educativas inclusivas.

Por otra parte, tal como expresan los resultados, los estudiantes de pedagogía manifiestan cierta resistencia para trabajar con especialistas de otras áreas, por lo que es necesario formar al profesor en el trabajo multidisciplinar, condición fundamental en la sociedad actual y base para una educación de calidad.

Finalmente, es necesario motivar estudios similares que proporcionen información para la toma de decisiones, y que ayuden a reestructurar programas, actualizar contenidos, definir perfiles, revisar la vinculación con el medio escolar y reflexionar acerca de las propias creencias que limitan las expectativas que los profesores ponen en sus alumnos. 


\section{BIBLIOGRAFIA}

Becerril, S. (1999). Comprender la práctica docente. México: Editorial Plaza y Valdés.

Cardona, C. (2002). Intervención con alumnos de alto riesgo en el área de las matemáticas. Alicante: Editor Club Universitario.

Castejón, J. (2002). Unas bases psicológicas de la educación especial. Alicante: Editor Club Universitario.

Defior, S. (2000). Las dificultades de aprendizaje: un enfoque cognitivo: lectura, escritura, matemáticas. España: Editor Aljibe.

Friz, M. (2007). Propuestas Didácticas en Educación Matemática para el nivel Inicial. Universidad del Bío-Bío, Proyecto FDD 2006-11. Registro Propiedad Intelectual $\mathrm{N}^{\circ} 163866$, Chile: Universidad del Bío-Bío.

García, J. (2001). Dificultades de aprendizaje e intervención psicopedagógica. Barcelona: Editorial Ariel.

Gay, L. R. \& P. Airasian (2000). Educational research: Competencies for analysis and application. Upper Saddle River, NJ: Prentice-Hall.

Gilroy, P. (1993). El conocimiento profesional y el profesor principiante. En: Carr, W. (Comp.). Calidad de la enseñanza e investigación-acción. Sevilla: Editorial Diada.

González López, Ignacio (2006, diciembre). Dimensiones de evaluación de la calidad universitaria en el Espacio Europeo de Educación Superior. Electronic Journal of Research in Educational Psychology, 4(3), 445-468. Obtenido el 19 de agosto de 2007 en http://www.investigacionpsicopedagogica.org/revista/articulos/10/espannol/Art_10_134.pdf

Imbernón, F. (2002). La investigación educativa como herramienta de formación del profesorado. Barcelona: Editorial GRAO.

Latorre, M. (2004). Aportes para el análisis de las racionalidades presentes en las prácticas pedagógicas. Estudios Pedagógicos 30: 75-91.

Lozano Díaz, Antonia (2003, abril). Factores personales, Familiares y Académicos que afectan al fracaso escolar en la Educación Secundaria. Electronic Journal of Research in Educational Psychology 1 (1): 43-66. Obtenido el 19 de agosto de 2007 en http://www.investigacion psicopedagogica.org/revista/articulos/1/espannol/Art_1_4.pdf

Llinares, Salvador (1994). El Profesor de matemáticas. Conocimiento base para la enseñanza y desarrollo profesional. En Santaló y otros (eds.) La enseñanza de las matemáticas en la Educación Intermedia. Rialp. Madrid.

Ministerio de Educación de Chile (2006). Resultados nacionales prueba Sistema de Medición de la Calidad de la Enseñanza (SIMCE). Santiago de Chile.

Miranda Casas, A., C. Fortes y M ${ }^{a}$ D. Gil Gil (1998). Dificultades del aprendizaje de las matemáticas. Un enfoque evolutivo. Archidona, Málaga, Aljibe.

Ramírez, María José (2002). Perfil de rendimiento de Chile en la subescala de representación de datos TIMSS 1999. Estudios Pedagógicos 28: 69-88.

Ramírez, María José (2005). Actitudes hacia las matemáticas y el rendimiento académico entre estudiantes de Octavo Básico. Estudios Pedagógicos 31 (1): 97-112.

Sanhueza, S. (2006). Prácticas Pedagógicas en la Construcción de la identidad profesional. Tesis de Magíster no publicada para optar al grado de Magíster en Ciencias de la Educación, Universidad Católica de la Santísima Concepción, Concepción, Chile. 